\title{
Interférences et création
}

La « dynamique auteur-éditeur » dans le processus de création chez Sony Labou Tansi à partir de la comparaison entre Machin la Hernie et L'État honteux

Jean-Pierre Orban

\section{(2) OpenEdition}

\section{Journals}

Édition électronique

URL : http://journals.openedition.org/genesis/599

DOI : 10.4000/genesis.599

ISSN : 2268-1590

\section{Éditeur :}

Presses universitaires de Paris Sorbonne (PUPS), Société internationale de génétique artistique littéraire et scientifique (SIGALES)

\section{Édition imprimée}

Date de publication : 30 octobre 2011

Pagination : 29-42

ISBN : 978-2-84050-804-5

ISSN : 1167-5101

\section{Référence électronique}

Jean-Pierre Orban, «Interférences et création », Genesis [En ligne], 33 | 2011, mis en ligne le 23 octobre 2013, consulté le 20 avril 2019. URL : http://journals.openedition.org/genesis/599 ; DOI : 10.4000/ genesis.599 


\title{
Interférences et création La « dynamique auteur-éditeur» dans le processus de création chez Sony Labou Tansi à partir de la comparaison entre Machin la Hernie et L'État honteux
}

\author{
Jean-Pierre Orban \\ J'écris énormément dans la tête, la nuit, le jour, avant que ça ${ }^{1}$ [fasse partie des] \\ littératures du point et de la virgule, c'est-à-dire là où il y a des phrases, \\ où il y a des papiers, où il y a un éditeur ${ }^{2}$. \\ Sony Labou TANSI
}

\begin{abstract}
À l'automne 1979, le Congolais (Congo-Brazzaville) Sony Labou Tansi (1947-1995), un des écrivains africains les plus puissants de sa génération, soumet son deuxième manuscrit de roman aux Éditions du Seuil. Elles le publieront en mai 1981 sous le titre L'État honteux. Entre ces deux dates, à la suite d'une demande de la maison d'édition, l'auteur aura remanié considérablement son manuscrit. En 2005, dix ans après le décès de Sony Labou Tansi, les Éditions Revue Noire ont fait paraître une version du manuscrit original intitulée Machin la Hernie. L'étude comparative des deux états du texte nous permet, loin de la suspicion qui, dans la littérature francophone, a longtemps pesé sur les études de cas semblables, d'étudier en quoi une intervention éditoriale peut influer sur le processus de création littéraire. En d'autres termes, d'analyser, sans préjugés, ce que l'on peut appeler la «dynamique auteur-éditeur».
\end{abstract}

$\mathrm{L}$ a conception moderne, occidentale et davantage encore européenne de l'auteur est solipsiste. L'auteur est tenu comme instance autonome de création et de production. Certes, de Taine à Bourdieu, les études n'ont pas manqué sur les déterminations sociales, économiques et culturelles qui conduisent l'auteur à être, ainsi que l'exprime Pierre Bourdieu à propos de Flaubert, " produit par ce qu'il contribue à produire3 », en d'autres termes à être objet plutôt que ou avant d'être sujet du processus de création. $\mathrm{Peu}$, en revanche, se sont intéressés au rapport intersubjectif qui peut exister dans ce processus entre l'auteur et les individus ou instances susceptibles d'intervenir dans sa création : époux, compagnes ou compagnons, proches, pairs, collaborateurs avoués ou non, institutions culturelles ou/et administratives, milieux ou structures avec lesquels l'auteur entretient des liens de fidélité ou d'allégeance, l'intermédiaire ou le «passeur" ${ }^{4}$ ou

\footnotetext{
1. Entretien de Sony Labou Tansi avec Bernard Magnier, cité dans J.-M. Devésa, Sony Labou Tansi, écrivain de la honte et des rives magiques du Kongo, Paris, L'Harmattan, 1996, p. 95.

2. Conférence au département des Sciences du langage et des littératures modernes comparées de l'université de Turin, 3 décembre 1986, ibid., p. 105, note de bas de page.

3. Pierre Bourdieu, Les Règles de l'art, Paris, Éditions du Seuil, coll. «Libre examen », 1992, 4e de couverture.

4. Voir ainsi les rapports entre Violette Leduc et Simone de Beauvoir évoqués par Catherine Viollet, «De la censure éditoriale à l'autocensure : Violette Leduc », dans Catherine Viollet et Claire Bustarret (éds), Genèse, censure, autocensure, Paris, Éditions du CNRS, 2005, p. 181-200.
} 
encore, dans une pratique culturelle moins latine qu' anglo-saxonne ou nordique, les ateliers professionnels d'écriture ou les échanges entre auteur et public à l'occasion de lectures de l'œuvre en cours d'écriture.

Parmi les instances d'interférence, il en est une particulièrement importante parce qu'elle représente une étape incontournable de la connaissance publique de l'œuvre, et parce qu'elle cristallise, en les reflétant ou en les anticipant, nombre d'autres interférences, attentes ou interdits. Il s'agit de l'éditeur. Passage obligé de l'œuvre littéraire, l'éditeur est à la fois une instance discrétionnaire (de jugement), de médiation entre l'auteur et le public et, dans une certaine mesure, variable selon les auteurs, les œuvres, les éditeurs et les zones culturelles, de coproduction de l'œuvre en ce qu'il peut la transformer en aval, la susciter et l'orienter en amont. Une instance qui a aussi la particularité, contenue dans la double signification du mot « éditeur », d'être à la fois une institution (l'éditeur en tant qu'organisme ou entreprise) et une personne (l'éditeur, référent de l'auteur au sein de la maison d'édition).

Dans ce rapport à l'instance éditoriale, l'auteur dit « francophone » et plus particulièrement l'auteur originaire du continent africain peut ou a pu présenter une spécificité. Cette spécificité provient ou proviendrait - car il s'agirait de la confirmer au-delà des a priori et des rumeurs - d'un décentrage par rapport aux lieux d'édition français et, plus précisément encore, parisiens.

Décentrage accentué si l'on remonte le temps et l'on considère le rapport entre colonie et métropole. Décentrage doublé aussi, dans ce cas, d'une dimension politique et sociale de domination/soumission réelle et consciente pour l'auteur colonisé. Un rapport dont on peut ensuite se demander s'il persiste, a persisté ou non, de façon consciente ou inconsciente, chez les écrivains postcoloniaux et si oui, jusqu'à quel moment et à quel point.

Propriété et propriétés de la langue
La spécificité de l'auteur francophone dans sa relation avec l'instance éditoriale passe indubitablement par un troisième terme qui est la langue. Et plus précisément, par une dialectique entre propriété et propriétés de la langue, entre son détenteur proclamé ou supposé et son utilisateur. $\mathrm{Si}$, par sa position géographique, culturelle ou sociale (voire linguistique pour les auteurs africains souvent, au moins, bilingues), l'utilisateur de la langue n'en est pas le propriétaire, doit-il, d'autant plus, en respecter les propriétés ? Et jusqu'où peut-il mettre en question, renouveler, déformer, détourner les règles sans être accusé soit d'ignorance soit de crime de lèse-majesté ? À l'inverse, s'il se conforme trop à la langue et, plus largement, au modèle non seulement linguistique mais aussi stylistique ou narratif en vigueur au centre, à une époque donnée, il court le risque de se faire accuser de manquer d'originalité.

C'est dans cette marge, souvent étroite, qu'opère l'auteur francophone de la «périphérie » et qu'il développe, consciemment ou non, une stratégie d'expression, mais aussi d'approche ou de conquête du « centre ». S'il existe ou a existé une particularité de l'auteur francophone - et particulièrement de l'auteur africain - c'est dans cette relation triangulaire auteur-éditeurlangue (ou modèle) et dans ce qu'elle implique de domination, de résistance, de compromis 
et de recréation ${ }^{5}$ que la probabilité de la trouver est la plus forte. Mais si c'est le cas, l'analyse de ce rapport n'est jamais loin de la suspicion qui a longtemps imprégné et imprègne encore les relations auteuréditeur et les commentaires à leur propos.

Suspicion de la part de l'auteur d'un rejet pour cause de non-conformité à un «modèle-propriétaire » qui lui serait imposé et qu'il ne pourrait pas remettre en question6. Suspicion, à l'inverse, du récepteur, éditeur, critique ou lecteur, d'une trop grande conformité au modèle et reproches de plagiat quand il y a réappropriation du modèle, comme cela s'est produit pour Yambo Ouologuem 7 , ou de réécriture forcée de l'œuvre, comme cela a été avancé pour Sony Labou Tansi ${ }^{8}$.

Une fois installée, cette suspicion semble rebondir sans fin. Car l'accusation de plagiat relance automatiquement la question de la propriété du modèle, celle-ci enclenchant la question de la possibilité ou de l'impossibilité de sa réappropriation, quand elle ne se double pas d'implications politiques. Elle pollue dès lors toute analyse de la relation auteur-éditeur en l'insérant dans un schéma d'affrontement parfois émotif, souvent irrationnel.

Une étude, nécessaire, de la relation entre auteur « francophone », spécialement africain, et éditeur «central » ne nous semble pouvoir se développer que si elle est dépassionnée. Cette «dépassionnalisation » ne peut être obtenue qu'à condition d'opérer une « réduction » de type husserlien. À savoir : 1) si l'on revient des opinions, rumeurs et $a$ priori vers les documents eux-mêmes ; 2) si l'on met entre parenthèses l'opposition frontale de deux protagonistes qui représenteraient de manière manichéenne l'un le créateur, l'autre le formateur (au sens où il «formaterait » l'œuvre du premier) ; 3) si l'on envisage, à l'inverse, la relation auteur-éditeur comme une intentionnalité réciproque (pour reprendre un terme husserlien), une dynamique, c'est-à-dire un processus en mouvement où des forces se combinent, s'allient, parfois s'opposent en un rapport intersubjectif constitutif du texte-livre.

5. On songe, notamment, à la translation du vocabulaire et de la syntaxe malinké dans la langue française opérée par Ahmadou Kourouma dans ses romans.

6. Voir, entre autres, la lettre manuscrite du 19 novembre 1948 de Kateb Yacine à Paul Flamand, directeur du Seuil, Littératures francophones au Seuil, une histoire, 1945-2006, Paris, Éditions du Seuil, 2006, p. 26.

7. Accusations de plagiat par rapport à André Schwarz-Bart (Le Dernier des Justes, Paris, Éditions du Seuil, 1959) et par Graham Greene (It's a Battlefield, London, Heinemann, 1952 ; C'est un champ de bataille, Paris, Laffont, 1953) à l'encontre de Yambo Ouologuem et son roman Le Devoir de violence (Paris, Éditions du Seuil, 1968).

8. Voir, entre autres, J.-M. Devésa, Sony Labou Tansi, op. cit. 


\section{Maïeutique de la réécriture}

Nous voudrions appliquer cette approche à l'étude comparative de L'État honteux 9 (EH), deuxième roman paru de l'écrivain congolais Sony Labou Tansi et de Machin la Hernie 10 (MLH), version antérieure du même roman parue dix ans après la mort de l'auteur, en 2005.

La place manque ici pour entrer dans le détail de l'argumentation, mais on peut, à défaut de posséder une version intermédiaire qui en constituerait, selon Labou Tansi lui-même, un «montage 11 », considérer le texte daté, sur le tapuscrit, du 5 juin 1979 et publié en 2005 sous le titre Machin la Hernie 12 comme la version de base du roman. Une version, éventuellement « remontée », que Sony Labou Tansi cherche, à la fin du mois de septembre 1979, à faire parvenir au Seuil.

Six mois plus tard, on sait que le Seuil l'a lue et a demandé à l'auteur de la « raccourcir ». Labou Tansi l'écrit dans une lettre du 27 mars 1980. Il s'apprête à partir le 6 avril aux États-Unis pour un voyage d'un mois organisé pour des personnalités africaines ${ }^{13}$. Il «fera » ce travail de réduction. « Pour la longueur, ajoute-t-il, c'est 320 pages de manuscrit interligne 2. Je vais ordonner tout ça en 200 pages (1/3 à flanquer ailleurs mais pas à l'eau) $)^{14} »$.

L'opération de « réduction » est entièrement effectuée entre le 7 avril, date d'arrivée aux États-Unis, et le 1er mai 1980, date ajoutée par Labou Tansi à la fin de la version éditée du roman ${ }^{15}$. Le témoignage de Yao Edo Amela, autre participant de ce voyage américain, à qui l'auteur codédiera le roman, le confirme ${ }^{16}$. Ensuite, selon le même témoignage, la nouvelle version du roman est remise au Seuil au retour de leur voyage.

Suit une longue période de dix mois, durant laquelle le texte demeure au Seuil sans décision de la part de l'éditeur. Une lettre de Labou Tansi non datée, mais écrite durant cette période en témoigne : «As-tu vu Luc Estang17 ? [...] Faut pas qu'il m’oublie deux ans. [...] S'ils ont des hésitations je mettrai le truc ailleurs $18 »$.

Enfin, le 3 février 1981, une lettre de Labou Tansi annonce qu'il « reste au Seuil et [que] le bouquin sort en mai [...]. Youpi !!!19».

9. Paris, Éditions du Seuil, 1981.

10. L'Atelier de Sony Labou Tansi, éd. établie par Nicolas Martin-Granel et Greta Rodriguez-Antoniotti, vol. III, Paris, Revue Noire, 2005.

11. «Je suis en train de monter et je crois que c'est bien le mot : L'État Honteux », lettre du 28 août 1979, ibid., vol. I, « Correspondance », p. 244.

12. Après que le Seuil a refusé aux Éditions Revue Noire de le publier sous le titre L'État Honteux (avec un H majuscule), écrit de la main de Sony Labou Tansi en tête du tapuscrit original, pour éviter que ne se retrouvent dans les rayons des librairies deux versions sous le même titre.

13. Yao Edo Amela, «Sony Labou Tansi, l’Amérique et moi », Centre d'étude d'Afrique noire (CEAN), n 65, Bordeaux, 2000, p. 31-39.

14. L'Atelier de Sony Labou Tansi, op. cit., « Correspondance», p. 249.

15. « Sugar-Hill, le 1er mai $1980 »$, L'État honteux, op. cit., p. 157.

16. «Sony Labou Tansi, l’Amérique et moi », op. cit., p. 33.

17. Éditeur du Seuil en charge de Sony Labou Tansi, au moins pour les premiers romans, avant d'être relayé par Gilles Carpentier : voir Littératures francophones au Seuil, op. cit., p. 47-50. Confirmé par les entretiens de l'auteur du présent article avec Jean-Marie Borzeix, ancien directeur littéraire du Seuil et avec Louis Gardel, conseiller littéraire au Seuil (respectivement le 28 mai 2010 et le 1er juin 2010).

18. L'Atelier de Sony Labou Tansi, op. cit., « Correspondance », p. 252.

19. Ibid., p. 253. 
L'État honteux paraît effectivement, la date du dépôt légal l'atteste, au deuxième trimestre 1981, plus précisément en mai 20 .

La question qui surgit alors est celle-ci : le texte soumis par Sony Labou Tansi à son retour des États-Unis, après réduction et, on le verra plus loin, réécriture, a-t-il été remanié par le Seuil avant publication douze mois plus tard?

Entre mai 1980 et début février 1981, l'écrivain congolais ne semble avoir aucune nouvelle de l'avis du Seuil. Celui-ci tombe comme une bonne surprise quelque temps avant le 3 février. Il n'a vraisemblablement aucune connaissance non plus d'un « retravail » éventuel du Seuil sur son texte. Or, contrairement à ce que les rumeurs laissent entendre, jamais, selon ses éditeurs ${ }^{21}$, le Seuil n'a imposé une modification du manuscrit sans l'accord de son auteur (on devrait préciser : du vivant de l'auteur, les œuvres posthumes faisant, à l'évidence, l'objet d'autres règles, comme l'indique l'exergue du Commencement des douleurs ${ }^{22}$ paru après la mort de Sony Labou Tansi : « Ouvrage édité par Gilles Carpentier »).

Cette règle, Jean-Marie Borzeix, directeur littéraire du Seuil de 1977 à 1984, à la tête d'une équipe d'une quinzaine d' « éditeurs », dont Luc Estang, l'affirme avec force : « $\mathrm{Au}$ Seuil, la règle était de faire des propositions à l'auteur. Il ne s'agissait que de suggestions, auxquelles on demandait à l'auteur de réagir23. » S'il reconnaît que Luc Estang a beaucoup travaillé avec Labou Tansi pour La Vie et demie, " des semaines, des mois ensemble », et témoigne que l'auteur congolais « était très à l'écoute de ce qu'on lui disait et en tenait compte ", qu'il était très intéressé à travailler avec un écrivain reconnu comme Luc Estang, auteur et éditeur classique aux antipodes de lui-même, qu'il a beaucoup appris avec lui, J.-M. Borzeix insiste sur le fait que rien n'était modifié sans un travail en commun ou une approbation par l'auteur des suggestions de l'éditeur.

Louis Gardel, auteur et conseiller littéraire au Seuil, confirme, à son tour, le témoignage de J.-M. Borzeix : «Luc Estang n'a jamais touché une virgule sans l'avis de l'auteur. » Luimême parle d'une relation «socratique » entre l'éditeur et l'auteur, une sorte de maïeutique pour faire « accoucher l'auteur de ce qu'il a dans les tripes », d'un « jeu à deux » dans lequel, veut préciser Louis Gardel, «l'auteur est le plus important».

Entre mai 1980 et février 1981, durant la période de «latence » au Seuil, il est possible que le tapuscrit ait fait l'objet de remaniements de la part de la maison d'édition. Mais il ne semble pas, à la lecture de la correspondance et, en particulier, de la lettre de Sony Labou Tansi du 3 février 1981 qui suit sans doute de près l'annonce de l'acceptation du tapuscrit par le Seuil, que l'auteur ait été mis au courant de changements à faire ou suggérés. Or s'il est vrai qu'aucune modification n'est effectuée sans l'accord de l'auteur, cela signifie que s'il y en a, elles sont annoncées avec l'avis d'acceptation du Seuil. Dans la lettre du 3 février, la date de parution est annoncée : elle aura lieu, dit Labou Tansi, en mai. Et c'est effectivement ce qui se passe. Trois mois ou trois mois et demi après l'annonce, ce qui laisse peu de place pour des remaniements de fond, et, difficilement, pour un travail en commun entre l'éditeur et l'auteur à une époque (1981) où les textes devaient être composés (sinon typographiés) avant d'être corrigés et renvoyés à l'auteur pour correction et bon à tirer. Par ailleurs, le fait

20. Achevé d'imprimer («5-1981»), 4e de couverture de L'État honteux, op. cit.

21. Entretiens avec J.-M. Borzeix et L. Gardel.

22. Paris, Éditions du Seuil, 1995.

23. Entretien du 28 mai 2010. 
que la date de parution est annoncée trois mois avant et est respectée semble indiquer qu'au moment de l'annonce, il n'est pas prévu de remaniement substantiel qui aurait empêché de prévoir avec une telle précision une date de parution.

Cela veut-il dire que le texte « américain » de Sony Labou Tansi n'a pas subi de modifications ou de propositions de modifications de la part de l'éditeur ? Non, bien sûr. Ces modifications ont peut-être été envoyées avec l'avis ou peu après. Mais on imagine mal l'éditeur envoyer une mouture remaniée et « ficelée », que l'auteur aurait simplement eu à accepter ou refuser.

S'il y a modification du texte par le Seuil, il est donc vraisemblable qu'il s'agit d'interventions qui ne demandaient pas de longues discussions ou d'aller-retour entre l'auteur et l'éditeur. En revanche, on peut faire l'hypothèse que si les interventions de l'éditeur n'ont pas été majeures, c'est que l'essentiel du travail de remaniement avait été réalisé au préalable par Sony Labou Tansi lui-même, entre la version qui a été nommée Machin la Hernie et la version publiée de L'État honteux. Peut-être lors de la version « montage » d'août 1979 pour ce qui est de l'agencement des épisodes. Mais plus vraisemblablement lors de ce que le compagnon de voyage de Labou Tansi aux ÉtatsUnis, Yao Amela, a appelé la «parturition » du roman. En réalité, une « reparturition », puisque l'auteur avait déjà accouché une première fois de son roman. Un deuxième accouchement donc, qui correspond au phénomène maïeutique dont parle Louis Gardel. L'histoire, ici, ne bégaie pas. Ce n'est pas une palilalie comme dans le roman lui-même. L'histoire se redit.

Et elle se redit, sans doute, avec les outils que Sony Labou Tansi a acquis lors de son travail en commun avec Luc Estang pour La Vie et demie. De fait, au niveau de la structure, la version finale de L'État honteux ne diffère pas fondamentalement de celle du premier roman, La Vie et demie.

Maïeutique, parturition, réaccouchement : la relation auteur-éditeur sur le «métier » luimême de remaniement du texte s'apparente à une dynamique où les forces ne s'opposent pas ou rarement, mais s'épousent. Ce qui se passe alors est une sorte de fécondation (in folio, ou « in libro ») et le travail qui s'effectue devient un vrai travail d'accouchement jusqu'à l'expulsion du texte-livre.

Dans le cas de L'État honteux, ce travail a été vraisemblablement opéré pour sa toute grande partie par l'auteur seul, mais à partir d'une injonction (à tout le moins d'une demande) de l'éditeur de réduire le texte d'un tiers. À partir de cette demande, tout semble indiquer que l'auteur s'attaque à une matière dont il ne supprime rien de substantiel, conformément à sa promesse de ne rien «flanquer à l'eau » mais que, dans un processus de type «transformiste 24 », il remodèle jusqu'à en faire un objet complètement autre.

24. Relevé par Nicolas Martin-Granel, notamment dans « Transcription, travestissement ou hypertexte ? Questions à propos de l'écriture de Sony Labou Tansi », dans Gilles Teulié (éd.), Les Littératures africaines : transpositions ?, Actes du colloque APELA, Montpellier 2001, coll. « Les carnets du CERPANAC », n², Université Paul Valery Montpellier III, 2002, p. 227-244. 
Comment s'opère cette remodulation ? Cette transformation en un autre que soi-même?

\section{Réduction quantitative}

La demande du Seuil consistait, selon Labou Tansi, à réduire d'un tiers le texte qui lui avait été soumis. En réalité, par rapport à Machin la Hernie, l'auteur va aller bien au-delà de cette requête.

En ajoutant au texte publié par les Éditions Revue Noire quinze pages tapuscrites que l'on sait disparues, on obtient quelque quatre cent dix mille signes. Le texte de L'État honteux édité au Seuil en comporte environ deux cent six mille. La « réduction » de l'un à l'autre est donc, non pas d'un tiers, mais de moitié.

$\mathrm{Au} v u$ des deux textes lus en parallèle, tout fait penser que le premier (MLH) a été

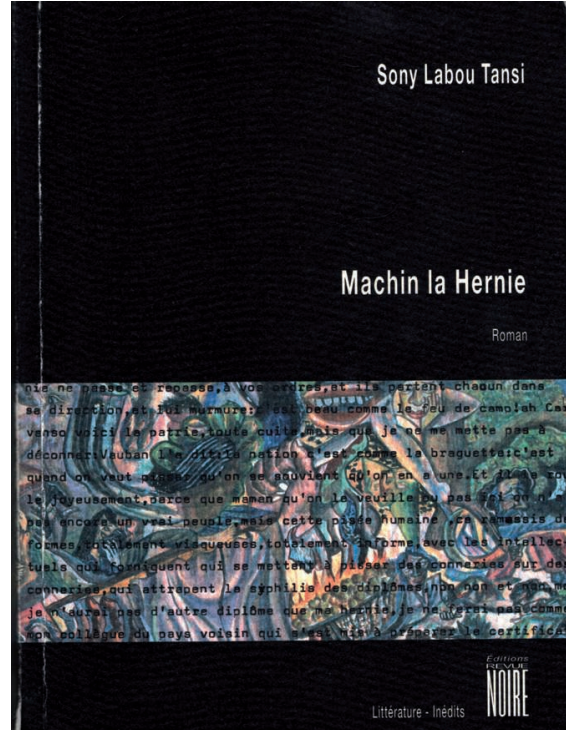
réécrit complètement en partant du début et en accentuant le processus de réduction au fur et à mesure de la progression dans la réécriture. $\mathrm{Si}$ on compare les « chapitres » (non numérotés) de L'État honteux aux sections équivalentes de Machin la Hernie, on constate en effet que la réduction en nombre de signes est nettement plus importante dans la deuxième partie que dans la première et que les deux derniers « chapitres » de L'État honteux sont le résultat d'une réduction de 75 à $80 \%$ de la matière initiale. Comme si l'auteur, pris par le temps à la fin du délai qu'il se serait accordé pour la réécriture (le séjour aux États-Unis), avait précipité le mouvement dans les derniers « chapitres ».

Et pourtant, malgré cette diminution drastique en termes quantitatifs et son accélération vers la fin du processus, il conviendrait mieux de parler de « contraction » que de réduction du texte initial. Car une étude comparée et pointilleuse des deux textes montre que du point de vue narratif, tout ce qui se trouve dans Machin la Hernie se retrouve dans L'État honteux. Selon le principe de Lavoisier soudain appliqué à la matière littéraire : « rien ne se perd, rien ne se crée, tout se transforme ».

Par quelles mutations?

En voici quelques-unes.

\section{Mutations qualitatives}

\section{I) DÉPlaCEMENTS}

Ces déplacements d'épisodes ou de blocs de texte plus ou moins inchangés ne semblent pas ou rarement répondre, comme la plupart des autres modifications, à une nécessité de structuration du texte ou d'ordonnancement du récit. Tout se passe comme si ces déplacements avaient été effectués avant même la «contraction » réalisée aux États-Unis, lors du «montage » d'août 1979 dont parle l'auteur congolais dans sa correspondance. Ou
De Machin la Hernie

à L'État honteux : rien ne se perd, tout se recrée

Fig. 2 : Page de couverture de Machin la Hernie (édition de 2005) 
comme si Sony Labou Tansi supprimait d'abord un passage avant de le reprendre plus loin, regrettant de l'avoir ôté ou le faisant remonter de sa mémoire où il avait toujours, ou depuis longtemps, été25. Selon un processus que lui-même évoquait et que N. Martin-Granel traduit par l'expression d'écriture mémorielle. Un passage de la mémoire, voire de l'inconscient à l'écriture consciente 26 après déplacement, transfert et recomposition. Ou d'une écriture « somnambulique » à une écriture « scripturaire »: «En fait, mes livres, je les écris d'abord dans ma tête... J'écris énormément dans la tête, la nuit, le jour, avant que ça vienne sur du papier $^{27}$. »

De Machin la Hernie à L'État honteux, on note plusieurs types de déplacements, du déplacement vers l'avant par bribes dans la même zone de texte ou de blocs dans d'autres zones, au rétrodéplacement, tous assortis de modifications plus ou moins profondes.

On ne peut donner ici qu'un seul exemple.

À la page 54 de Machin la Hernie, le protagoniste Martillimi Lopez, président d'un État africain, parle de Camizo Diaz, un traitre :

[...] je l'ai fait colonel comme tout le monde croyant qu'il allait remercier ma hernie, le monde est méchant, regardez-le mes frères et chers compatriotes, nu comme vous le voyez là, regardez-le du pied à la tête : qu'est-ce qu'il a de plus que ma hernie ? et le coin de la foule qui a toujours cherché midi à quatorze heures répond : la hernie ! et Lopez de maman national éclate de son rire de père, comment pouvez-vous croire une chose pareille $[\ldots]$

Suit une «suspension » de six pages non reprises dans L'État honteux à la suite du passage ci-dessus. Dans L'État honteux, le texte ci-dessus est repris avec d'autres mots :

[...] un Camizo Diaz, que je suis allé chercher au fin fond de la patrie, qui ne savait même pas manger un saucisson, je l'ai fait sergent, je l'ai fait capitaine puis colonel comme tout le monde, et voilà comment il me dit merci. Et regardez, mes frères et chers compatriotes, nu comme vous le voyez là : qu'est-ce qu'il a de plus que ma hernie ?

Le coin de la foule qui a toujours cherché midi à quatorze heures a répondu : « La hernie », et Lopez de maman éclate de rire [...] (EH, p. 50-51).

La reprise s'arrête à « éclate de rire ». Suit un passage qui constitue l'un des rares ajouts de L'État honteux, clôt le «chapitre » et a pour fonction de structurer la narration : «que l'enquête commence ». Mais le début de cet ajout («ça y est, je suis consolé ») est lui-même une reprise de la fin des six pages « suspendues » : «d'accord ma hernie est comblée » (MLH, p. 59) :

[...] : ça y est, je suis consolé : il donne l'ordre à Carvanso de couper l'engin de palabre du colonel Camizo Diaz, de le mettre dans le formol et de le faire placer dans ma chambre à côté du portrait de maman, juste au-dessous du portrait de feu ma femme Atélu-Léa, morte pour la patrie, pendue

25. Nicolas Martin-Granel parle d'« hypotexte oral toujours "déjà là" », dans N. Martin-Granel et G. RodriguezAntoniotti, «Approche génétique des écrits littéraires africains. Le cas du Congo », Dossier littéraire, dans Études littéraires africaines, $\mathrm{n}^{\circ} 15,2003$, p. 62.

26. Ibid., p. 37-38.

27. Entretien de Sony Labou Tansi avec Bernard Magnier, passage cité en exergue du présent article. 
par les cons de leurs mamans qui ont voulu me faire croire qu'elle s'est pendue mais comment pendue ? et je vous en prie, que l'enquête commence (EH, p. 51).

Quant aux six pages suspendues, leur texte en est repris par bribes parfois à des endroits très distants de l'original (ainsi de MLH, p. 54 à EH, p. 156), mais plus souvent dans une recomposition au chapitre suivant de L'État honteux. Ainsi :

[...] qu'est-ce que vous alliez devenir si j'avais été [suivent quarante-trois lignes non reprises dans $\left.l^{\prime} E H\right]$ Tarvanso national qui convoquait le conseil de la nation pour statuer sur le cas honteux de mon cousin Jazo Portes Luna qui a dormi avec la femme de mon autre cousin Argento Comma [...] (MLH, p. 54-55).

Qu'est ce que vous auriez fait si j'avais été Yao Tananso qui convoquait le Conseil de la nation pour des conneries de mon cousin Zozo Portes Luna qui a « dormi » la femme de mon autre cousin Argento Comma? (EH, p. 52).

Cet exemple, parmi des dizaines d'autres possibles, montre bien les modifications opérées dans la " condensation-réécriture » du texte. Le texte est repris à d'autres emplacements parfois très éloignés les uns des autres. Le puzzle des phrases est recomposé mais très rarement à l'identique. Les noms des personnages (mais c'est vrai aussi des noms de lieux) sont modifiés, souvent sans raison, sauf ici pour Jazo en Zozo, plus signifiant.

À noter que les deux derniers extraits tendent à prouver que la réécriture, en tout cas ici, est bien le fait de l'auteur lui-même. Car pourquoi l'éditeur passerait-il de Tarvanso à Tananso (le prénom Yao est nouveau et renvoie au compagnon de Labou Tansi aux USA) ? Pourquoi et comment introduirait-il dans la nouvelle version une expression familière du Congo, «dormir » une femme sauf, dans un acte de folie prométhéenne, à se prendre pour le créateur lui-même du texte ? D'autant que le texte réécrit est moins soutenu et moins harmonieux : «qui convoquait le Conseil de la nation pour des conneries de mon cousin Zozo Portes $^{28}$ » au lieu de « qui convoquait le conseil de la nation pour statuer sur le cas honteux de mon cousin Jazo Portes ».

\section{2) AJOUtS NOTABLES (REPÈRES NARRATIFS, SPATIAUX, TEMPORELS)}

On sait que Sony Labou Tansi ne retravaillait pas ses textes par ratures mais par recommencements ${ }^{29}$ en remodelant la matière elle-même, comme une masse de glaise, et plutôt en «surabondant» qu'en élaguant. On le constate pour ce roman aussi. Mais pas pour la « surabondance ».

Entre Machin la Hernie et L'État honteux, il y a en effet très peu d'ajouts, sauf de détail, au sein d'un paragraphe ou d'une phrase. En revanche, on trouve un certain nombre - six ou sept - d'ajouts notables ou significatifs qui ont, pour fonction ou, à tout le moins, pour résultat essentiel d'introduire des repères narratifs, temporels et spatiaux. Ils se trouvent significativement au début (reprises de la trame ou reprise en main du récit par le narrateur pluriel) ou à la fin d'un chapitre (introduction d'une chute et/ou appel de la suite du récit).

28. C'est nous qui soulignons, ainsi qu'à chaque fois qu'un soulignement apparaît dans une citation.

29. N. Martin-Granel et G. Rodriguez-Antonietti, «Approche génétique des écrits littéraires africains », op. cit., p. 61 . 
Un seul de ces ajouts va nous servir d'illustration.

Machin la Hernie se termine par le retour précipité d'Europe de Martillimi Lopez après qu'il a été averti d'une tentative de coup d'État par Vauban, son conseiller de sécurité blanc et par ailleurs homosexuel. Le potentat reprend le pouvoir, fait arrêter Vauban et le fait servir à manger lors d'une réception où il invite les représentants et chefs d'État étrangers. Derniers mots du récit : «Prenez et mangez, ceci est Vauban. »

La fin de L'État honteux reprend cet épisode et cette parole, mais les fait suivre d'un discours de contrition de Martillimi Lopez : «non, non et non, moi, Lopez [...] je dis : terminés les jeux de hernies [...]. Je rends le pouvoir aux civils» (EH, p. 157). Mais elle les fait surtout suivre par la reprise de la narration par un narrateur pluriel, le «nous » populaire qui apparaît de temps en temps dans Machin la Hernie mais beaucoup plus dans L'État honteux, un «nous » qui ramène Martillimi Lopez « à Moumvouka, le village de MamanFolle-Nationale », comme il(s) l'avai(en)t fait « quarante ans auparavant ».

Cet épisode ${ }^{30}$ permet d'encadrer temporellement (quarante ans) et spatialement (retour au village) toute l'histoire du pouvoir de Martillimi Lopez que raconte le roman nettement plus narratif qu'est L'État honteux. En effet, au début de ce dernier (à défaut de connaître l'incipit de Machin la Hernie), on peut lire :

Voici l'histoire de mon-colonel Martillimi Lopez [...]. Nous le conduisîmes du village de Maman Nationale à la capitale où il n'était jamais venu avant, jamais de sa vie. Nous le conduisîmes au milieu des chants, des salves de canons, des vivats et des cris ; lui chantait l'hymne national, assis sur le dos de Moupourtanka son cheval blanc. [...] Nous étions tous sûrs que cette fois rien à faire nous aurions un bon président. Nous portions [...] son seau hygiénique [...] ses trois caisses de moutarde Bénédicta [...] (EH, p. 7-8).

Du début à la fin de L'État honteux, la boucle est bouclée : le président est renvoyé chez lui, avec son seau et ses pots de moutarde, et les tirailleurs qu'il découvre à son arrivée à la capitale sont renvoyés dans leur caserne.

\section{3) Segmentation en chapitres, alinéas, PHrases}

La structuration narrative du roman se poursuit dans celle de sa forme.

- Resegmentation en chapitres

Machin la Hernie comporte trois parties, numérotées en chiffres romains. Ces divisions, dont on ne voit pas bien à quelles divisions du récit lui-même elles correspondent et de longueur assez déséquilibrée (50 p., 85 p. et 145 p.) semblent plutôt représenter le mouvement d'une écriture qui s'accélère et s'amplifie avec les pages.

Face à Machin la Hernie, L'État honteux n'est plus divisé en parties mais en neuf « chapitres ». Leur longueur grandit progressivement jusqu'aux trois quarts du livre avant de diminuer brusquement : « chapitre $» \mathrm{I}=8 \mathrm{p} . ; \mathrm{II}=9 \mathrm{p}$. ; III $=10$ p. ; IV $=18 \mathrm{p} . ; \mathrm{V}=10$ p. ; $\mathrm{VI}=30 \mathrm{p} . ; \mathrm{VII}=38 \mathrm{p}$; $\mathrm{VIII}=15 \mathrm{p}$. ; IX $=13 \mathrm{p}$. Comme on l'a constaté en termes de signes typographiques (voir section I), l'écriture s'amplifie avant de faiblir et de se contracter.

30. Outre qu'il permet de ne pas terminer sur un banquet anthropophagique qui renvoie de façon trop évidente, il nous semble, à la fin de la partie IV de L'Automne du patriarche de Gabriel Garcia-Marquez (Paris, Grasset, 1976, p. 148). 


\section{MACMIN LA HORNEE}

ce lit présidentiel où ma petite dort un sommeil du tonnerre, il s'endort tout habillé, chaussé, boueux de cette boue amère où mon peuple piétine, il dort sur ses soixante-quinze médailles de ma guerre contre la Russie, les poings fermés, la braguette ouverte, comme un vra crocodile de boue, puant la bière et l'aubergine, il ronfle, ne se retournera que le matin pour prendre ma décision de me marier dans cette tenue peinte aux couleurs réelles de mon peuple, mon colonel Carvanso qui rentre de mission essaye de l'en dissuader : les blancs vont se moquer monsieur le président, ils vont se moquer et la presse va en profiter, mais Carvanso les blancs peuvent se moquer, ils ont eu Louis oui Louis XIV qui ne se lavait qu'une fois tous les sept ans, et il entre dans son rire d'historien pour parler de Vauban qui ne se lava que quelque sept fois pendant une vie comme celle de Vauban, mais monsieur le président vous avez de la boue dans les oreilles, vous en avez dans les narines, vous en avez dans les cheveux, et t'en fais pas Carvanso, c'est une boue historique, elle vient de mon peuple, elle sent le peuple, et il la roule.

Il marche tout boueux devant les délégations et leurs chefs, il leur serre la main en leur laissant un peu de sa boue historique, il la serre à sa majesté de Belgique qu’il embrasse à la manière des ancêtres, laissant un peu de sa crasse historique sur les revers de son habit royal, il la serre à sa majesté princesse de Danemark qu'il embrasse à la manière des ancêtres en lui laissant de sa crasse historique, il embrasse tous les amis de mon peuple à la manière de mon peuple en leur laissant sur la poitrine

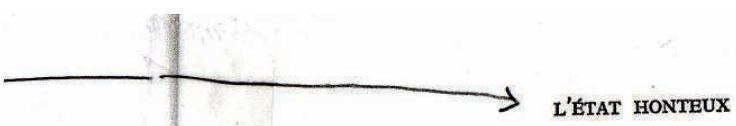

46

de fauve national, il lui a laissé ses dents blanches et sa barbiche. Puis il vient se jeter tout habillé sur son lit présidentiel, dort son sommeil de lion, tout chaussé, tout boueux, il dort sur les soixante-quinze médailles de ma guerre contre les communistes, les poings fermés, la braguette ouverte, comme un vrai caïman de boue, les dents dehors, la main droite sur son arme, puant la bière à l'aubergine, il ronfle.

- Je veux me marier dans cette tenue.

Carivanso national essaye de l'en dissuader:

- Monsieur le Président les Blancs vont se moquer. Ils vont se moquer. La presse va en profiter. - Mais, Carvanso, les Blancs peuvent se moquer: ils ont eu Louis XIV qui ne se lava que quelque six fois dans une vie comme celle de Louis XIV, et Vauban, et Frédéric II_Il entre dans son rire d'historien pour parler de Catherine de Russie qui...

- Monsieur le Président vous en avez plein les narines, plein les oreilles et je suis sûr qu'ils vont se moquer.

- C'est la boue de mon peuple : qu'ils se moquent. L'Afrique doit rester l'Afrique. Oui, elle doit remettre le monde au monde.

Il marche tout boueux devant les délégations et leurs chefs. Tout le monde applaudit. Il serre la main à Sa Majesté des Flamands qu'il embrasse à la manière des ancêtres en lui laissant un peu de sa boue historique; il la serre à Sa Majesté princesse de Danemark qu'il embrasse à la manière des ancêtres en lais-

Les débuts de chapitre n'indiquent pas nécessairement mieux que dans Machin la Hernie le début d'un nouvel épisode ou d'un nouveau sujet. En revanche, les chutes des chapitres sont plus marquées. Et ces chutes sont souvent des ajouts. Fin du « chapitre » III : " "Taisonsnous, Papa National est en train d'aimer sa femme." Pas de musique. Pas de voitures. Personne dans la rue. Pendant deux jours »; fin du chapitre IV : « que l'enquête commence »; fin du chapitre $\mathrm{V}:$ « terminé avec les plats de mon peuple, terminé avec les boissons de vos mamans par lesquelles vous avez essayé de m’avoir»; fin du chapitre VI : «Donnez-lui la leçon de ma hernie »; fin du chapitre VII : « je m'en vais les revoir »; fin du chapitre VIII : « vous me boufferez comme vous m'avez foutu ».

\section{- Division en paragraphes}

Cette opération est visible à l'œil nu. On passe d'un continuum, sans alinéa, sans guillemets (une seule occurrence dans Machin la Hernie), sans tirets de dialogue, sans points, sans capitales, sauf à « Dieu »... à une matière typographiquement et syntaxiquement (et même « visiblement ») restructurée.
Fig. 3 : Face à face de deux pages de Machin la Hernie et de L'État honteux, variantes l'une de l'autre 
- Ponctuation : insertion du point

Logiquement, dans ce mouvement de structuration typographique et syntaxique, la ponctuation acquiert une plus grande importance. Mais, à l'analyse, on se rend compte que la ponctuation existe dans Machin la Hernie, quasi toute la ponctuation, y compris, même s'ils sont rares, les guillemets et les deux-points. Le seul signe de ponctuation qui manque, en tout cas est rare dans Machin la Hernie, c'est le point. Comme s'il ne s'agissait pas tellement pour l'auteur d'un procédé stylistique mais plus d'un mouvement d'écriture qui ne veut pas s'arrêter, ou le moins possible. Les dialogues sont souvent marqués par des deux-points, ou des points-virgules, mais l'auteur ne veut pas mettre de point, de temps d'arrêt (au mieux des temps de suspension - des pauses - à la fin des « chapitres »). Il veut laisser se poursuivre le discours logorrhéique de Martillimi Lopez.

Dans L'État honteux, le point est introduit... et tout change. Quelques longues périodes existent encore (EH, p. 148-149), mais elles sont plus rares. La plupart des phrases sont courtes, typographiquement marquées. Les capitales sont rétablies pour les noms propres et leur utilisation est uniformisée. Les guillemets apparaissent pour les jeux de mots : ainsi « Flamants », avec capitale et guillemets pour désigner les Flamands.

- Introduction de dialogues typographiquement visibles (passage du style indirect libre au style direct)

Dans L'État honteux, les dialogues sont désormais indiqués par des alinéas et des tirets de dialogue. Machin la Hernie ne comporte que trois dialogues ainsi marqués, tous les trois apparaissant dans les six dernières pages du livre, comme si, arrivé à la fin de sa première étape de « jet », l'auteur s'apprêtait à entrer dans la deuxième phase d'écriture, à passer à la littérature, comme il le dit, « du point et de la virgule, c'est-à-dire là où il $\mathrm{y}$ a des phrases, où il $\mathrm{y}$ a des papiers, où il y a un éditeur ${ }^{31} »$. L'État honteux en compte des dizaines, parfois avec création de répliques simples, d'acquiescement : « — Oui, monsieur le Président. »

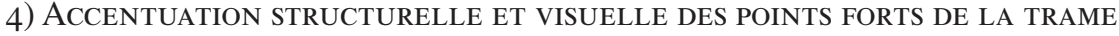
NARRATIVE

Tout au long de L'État honteux, sont insérés des repères pour les épisodes qui peuvent se révéler importants pour structurer la trame narrative, pour faciliter la lecture, assister le lecteur dans sa compréhension du texte, mais surtout transformer en récit ce qui est au départ un long flux de parole, un discours-fleuve incoercible.

Les épisodes tels que l'enquête, les meurtres, les mises à mort, faits marquants ou références aux grands temps du récit (EH, p. 61) sont isolés, en tout cas fortement repérables en étant placés en début ou fin de chapitre (EH, p. 32-33), par des blancs (EH, p. 113) ou dans des paragraphes commençant par des mots-clés.

Des éléments de liaison supplémentaires sont introduits, par exemple : «Le refrain revient » $(\mathrm{EH}$, p. 28$)$; «C'est à cette époque » $(\mathrm{EH}$, p. 132).

31. Conférence de Sony Labou Tansi au département des Sciences du langage et des littératures modernes comparées de l'université de Turin, passage déjà cité en exergue du présent article. 


\section{5) RÉÉCRITURE DU TEXTE EN MIROIR DÉFORMANT}

De la même manière que les grands segments narratifs sont repris ou déplacés dans la structure du récit, on observe, à l'intérieur de ces segments eux-mêmes restructurés, une reprise, parfois avec déplacements des mêmes éléments.

Déplacements-reprises, mais souvent avec transformations : utilisations de synonymes, modifications des noms de personnages (parmi des dizaines d'exemples : Barça Baldi en Berthe Salazar, Walansoen en Anzcox, Letanso Nanti en Letanso, Caranto Benito en Caranto Muhete, Agostino Lafonsai en Hugo de Lafundia, Vermoz Diaz en Yambo Yambi), changements de race d'un animal (Narka, le perroquet de Martillimi Lopez, devient un cheval en passant dans L'État honteux (MLH, p. 209-EH, p. 134) - ailleurs un autre (?) perroquet devient un chien (MLH, p. 138-EH, p. 90). Tout opère comme si le texte avait, presque au sens propre, muté. Ou comme si les deux écritures, celle de Machin la Hernie et celle de L'État honteux, étaient en miroir. Mais en miroir déformant.

Car dans ce mouvement de contraction, à l'intérieur du nouveau cadre ou moule imposé ou intégré à partir de la demande de l'éditeur (peut-être assortie de recommandations précises en matière de segmentation, de repérage, de création de dialogues, etc.), Sony Labou Tansi réécrit tout. Hormis deux ou trois passages (MLH, p. 169-EH, p. 120-121 ; MLH, p. 172173-EH, p. 124-125 ; MLH, p. 249-EH, p. 145) repris à l'identique, aucun extrait ne sort indemne du passage d'une version à l'autre.

La lecture comparative de Machin la Hernie et de L'État honteux laisse à penser que l'essentiel du travail de réécriture a été effectué par l'auteur lui-même. À partir de la demande de réduction formulée par l'éditeur, l'opération est devenue celle d'un ordonnancement d'une matière compacte, la canalisation d'un fleuve impétueux, sa dérivation vers des bassins circonscrits et sages. La mue d'une écriture poétique, voire « poématique 32 », ainsi que le dit Daniel Delas en empruntant le terme à Henri Meschonnic ${ }^{33}$, en une structure romanesque où est mis au jour le fil narratif.

Une «normalisation ${ }^{34} »$ ? Sans doute. Le passage d'une écriture dionysiaque, nocturne à un modèle apollinien selon des normes définies en un temps, un espace, une culture et un marché donnés.

On doit s'interroger sur ce modèle, et les créateurs le remettront en cause. Comme lecteur, on pourra regretter le «formatage » qui a abouti au texte final. On pourra, à l'inverse, juger ce dernier plus accessible que l'original, préférer à l'ancien le nouveau rejeton issu de la mutation génétique opérée dans le processus de contraction et de réécriture.

Mais ce jugement à la Salomon ne convient pas ici. Il ne s'agit pas de choisir. Si l'étude comparative des deux états de L'État honteux de Sony Labou Tansi a un seul mérite, c'est de montrer la nécessité de les avoir tous deux publiés.

32. Daniel Delas, « Métastases du discours postcolonial de Machin la Hernie à L'État honteux », dans Sony Labou Tansi à l' euvre, Actes du colloque APELA, Paris XII-Paris XIII, 2007, dir. Papa Samba Diop et Xavier Garnier, Paris, L'Harmattan, 2007, p. 65-73.

33. Henri Meschonnic, Hugo, la poésie contre le maintien de l'ordre, Paris, Maisonneuve et Larose, 2002.

34. Daniel Delas, « Métastases du discours postcolonial... », op. cit., p. 71.

\section{Écriture dionysiaque et modèle apollinien}


Jean-Pierre Orban, philosophe de formation (Université catholique de Louvain) et diplômé en communications sociales (Université libre de Bruxelles), dirige la collection «L'Afrique au cœur des lettres » (Paris, L'Harmattan) et a publié, à ce titre, plusieurs inédits manquant au patrimoine littéraire africain. Il est chercheur associé à l'ITEM (CNRS-ENS), au sein de l'équipe « Manuscrit francophone ».

Jean-Pierre Orban,jporban@wanadoo.fr

Résumés Interférences et création

Les interférences dans le processus de création littéraire ont été assez peu étudiées. L'article s'attache en particulier aux interventions éditoriales. Celles-ci s'avèrent sensibles dans la littérature francophone et plus spécifiquement chez les auteurs africains situés dans un rapport colonial ou postcolonial à l'édition française. La suspicion de réécriture forcée des manuscrits a longtemps régné dans ces rapports et a conduit aux rumeurs les plus diverses, souvent infondées. L'article entend proposer une étude dépassionnée, fondée sur des documents, de la relation auteur-éditeur conçue comme une dynamique plutôt que comme un affrontement. Dans cet esprit, sont comparées les deux versions connues du roman L'État honteux de l'écrivain congolais Sony Labou Tansi. Une analyse qui vise à démontrer que la réécriture de la première version est essentiellement le fait de l'auteur luimême mais à partir d'une recommandation expresse de l'éditeur, la réponse dépassant dans ce cas les termes de la demande.

Interferences in the literary creation process have rarely been studied. This article will focus in particular on editorial interventions. These turn out to be quite sensitive in Francophone literature and more specifically in the works of African writers who have a colonial or postcolonial relation with French publishing houses. The suspicion of forced rewritten manuscripts has long dominated these relationships and [has] led to various rumors, mostly groundless. This article, based on documents, presents a dispassionate study of the author-editor relationship understood as a dynamics rather than a confrontation. It is in this spirit that we will compare the two known versions of the Congolese writer Sony Labou Tansi's novel L'État honteux. This analysis aims to demonstrate that the rewriting of the first draft is due essentially to the writer himself but under the editor's imperative recommendations. The outcome in this instance goes far beyond the injunction.

Die Interferenzen im literarischen Schaffensprozess wurden von der Forschung bislang ziemlich wenig beachtet. Der Beitrag befasst sich hauptsächlich mit Eingriffen durch Verleger. Diese haben sich in der französischsprachigen Literatur als delikat erwiesen, insbesondere bei afrikanischen Autoren, die in einem kolonialen bzw. postkolonialen Bezug zu französischen Verlagen stehen. Der Verdacht des Umschreibens von Manuskripten unter Zwang besteht seit langem in diesem Bereich und hat zu den verschiedensten, oftmals unbegründeten, Gerüchten Anlass gegeben. Der Beitrag versteht sich als Vorschlag für eine sachliche, auf Dokumenten beruhende Untersuchungsweise des AutorVerleger - Verhältnisses, verstanden vielmehr als Dynamik denn als Konfrontation. In diesem Sinne werden die zwei bekannten Versionen des Romans L'État honteux des kongolesischen Autors Sony Labou Tansi verglichen. Die Analyse zielt darauf ab zu beweisen, dass die Umschrift der ersten Version, wenn auch auf ausdrückliche Empfehlung des Herausgebers, tatsächlich das
Werk des Autors selbst ist, dessen Antwort in diesem Fall weit über den Wortlaut der Forderung hinausging.

Las interferencias en el proceso de creación literaria han sido bastante poco estudiadas. Este artículo está centrado sobre todo en las intervenciones editoriales, particularmente sensibles en la literatura francófona y más específicamente en lo que respecta a los autores africanos, confrontados a una relación colonial o postcolonial con la edición francesa. La sospecha de una reescritura forzada de los manuscritos se impuso durante mucho tiempo en esas relaciones y produjo los rumores más diversos, a menudo infundados. Este artículo intenta proponer un estudio desapasionado, basado en documentos, de la relación autor-editor concebida como una dinámica más que como un enfrentamiento, y compara, en esta perspectiva, las dos versiones conocidas de la novela L'État honteux del escritor congolés Sony Labou Tansi, cuyo análisis demuestra que la reescritura de la primera versión es obra esencialmente del autor mismo, aunque esté originada por una recomendación explícita del editor. En este caso, la respuesta sobrepasa los términos de la solicitud.

Le interferenze nel processo della creazione letteraria sono state poco studiate. L'articolo concerne in particolare gli interventi editoriali. Questi si mostrano significativi nella letteratura francofona e più specificamente per gli autori africani che intrattengono un rapporto coloniale o postcoloniale con l'edizione francese. Il sospetto di riscrittura forzata dei manoscritti esiste da lungo tempo e ha dato luogo alle più diverse dicerie, spesso infondate. L'articolo propone un'indagine spassionata, fondata sui documenti, della relazione autore-editore, concepita come una dinamica piuttosto che uno scontro. In questo spirito sono comparate le due versioni conosciute del romanzo L'État honteux dell'autore congolese Sony Labou Tansi. Un'analisi che dimostra come la riscrittura della prima versione sia essenzialmente dell'autore, anche se da una raccomandazione esplicita dell'editore: un caso in cui la risposta sorpassa i termini della richiesta.

As interferências no processo de criação literária têm recebido pouco estudo. $\mathrm{O}$ artigo dedica-se em especial às intervenções editoriais. Estas revelam-se sensíveis na literatura de língua francesa e mais especificamente nos autores africanos situados em relação colonial ou post-colonial com a edição francesa. A suspeita de reescrita forçada de manuscritos reina há muito neste domínio e levou aos rumores mais diversos, geralmente infundados. $\mathrm{O}$ artigo propõe um estudo desapaixonado, fundado sobre documentos, da relação autor-editor concebida como uma dinâmica e não como uma confrontação. Neste espírito, são comparadas as duas versões conhecidas do romance L'Etat honteux do escritor congolês Sony Labou Tansi. Uma análise que visa demonstrar que a nova redacção da primeira versão é essencialmente devida ao autor, mas motivada por uma recomendação expressa do editor: neste caso a resposta excedeu os termos do pedido. 